Originalveröffentlichung in: Zeitschrift für Assyriologie und vorderasiatische Archäologie 74, 1984, S. 164-167

\title{
Zur Lesung einiger frühdynastischer Inschriften aus Mari
}

\author{
Von Manfred Krebernik - München
}

Die Mehrzahl der frühdynastischen Inschriften aus Mari wurde zusammenfassend - jeweils mit Textkopie - von G. Dossin in MAM 3, S. 307-331, veröffentlicht und bearbeitet. Ein Katalog der beschrifteten Statuetten findet sich bei E. A. Braun-Holzinger, Frühdynastische Beterstatuetten (1977) S. 69-72; beigegeben sind dort - neben der Bibliographie - Transkription (gegenüber der Erstpublikation mehrfach mit neuen Lesungen) und Analyse der jeweiligen Inschrift.

Im folgenden seien die Ergebnisse einer - unter dem Blickwinkel eines Vergleichs mit den Texten aus Ebla erfolgten Durchsicht dieser Texte kurz mitgeteilt.

1. LAM-gi $i_{4}-m a-r i$ (M. 174: Braun-Holzinger S. 69)

Der erste Teil des Königsnamens dürfte mit LAM $=i \check{s}_{\mathrm{x}}$, das auch in Ebla für häufiges LAM $\times$ KUR $=i \check{s}_{11}$ (auch aAK) vorkommt, als Verbalform $i \breve{s}_{\mathrm{x}}-g i_{4} \mathrm{zu}$ interpretieren sein, vgl. mit $i \breve{s}_{11}$-gi-gebildete Namen in Ebla ${ }^{1}$. Die zugrundeliegende Wurzel ist vielleicht $\check{s}^{-} q-y$ "tränken " 2 . Zu beachten ist schließlich, daß gi $i_{4}$ in Ebla nicht als Syllabogramm gebräuchlich ist ${ }^{3}$.

2. GUM.BÀD (M. 2239 Z. 1, M. 2278 Z. 3: MAM 3, Nr. 21 [S. 325] bzw. Nr. 12 [S. 319f.]; Braun-Holzinger S. 71)

Der PN könnte gum-dùr zu lesen sein. Durch Glosse ist dùr für Tell Abū Șalābīh bezeugt in dem PN $\Pi l-(t u r)_{\text {(tù }}{ }^{4}$. Mit gum begin-

1 Für solche Namen s. MEE 2, S. 326; ARET 2, S. 108; ARET 3, S. 288. Die dortigen Belege bieten auch Beispiele für den Wechsel $i \check{s}_{11} / i \check{s}_{\mathrm{x}}(\mathrm{LAM})-g i\left(\right.$ MEE $2, i \check{s}_{\mathrm{x}} "=i \check{s}_{11}$; ${ }_{n} \check{S}_{y}{ }^{\prime}=$ LAM). [S. a. J.-R. Kupper, RlA VI 446.]

2 šaqu „hoch sein" und $z a k \hat{u}$,rein sein" scheiden aus, da tertiae $w$. Bei šegûu „wüten" $\left(\check{s}-g-{ }^{-}\right)$würde man $a$-Vokalismus erwarten.

3 Vgl. Verf., ZA 72 (1972) 188.

${ }^{4}$ OIP 99 (=IAS) S. 35. dùr ist hier wohl $d \bar{u} r$ „Festung“ (vgl, die mit Anm. 8 belegten PN), nicht Imperativ /tūr/. 
nende PN sind in Ebla nicht selten ${ }^{5}$ - es handelt sich wohl um /qūm/ "steh auf!“. Daneben wäre dùr dann ein zweiter Imperativ, wohl (tūr/ „kehre zurück!“). Als Beispiele für eine solche Namenbildung lassen sich in Ebla Gum-zét und Gul-zét heranziehen, falls zé =/și/ „komm hervor!".

3. BE.BU.BÀD (M. 2241 Z. 6: MAM 3 S. 329 f.)

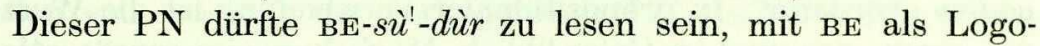
gramm für „Herr“, wie es auch in Ebla geläufig ist. Nach der Photographie ist reguläres sÙ anstelle von BU nicht auszuschließen. $\mathrm{Zu}$ dùr s.o. (2). Ähnliche PN aus Ebla sind BÉ.SÙ-KUR.KI, BE.SÙÉREN+X $\left(=\mathrm{LU}_{\mathrm{X}}\right)^{8}$.

4. "(G)ištarat" (M. 2241 Z. 9, M. 2248 Z. 4': MAM 3 S. $329 f$.)

Der GN wurde von Dossin als fem. Form von Ištar aufgefaßt und auf einen sum. Ursprung *Gišdar zurückgeführt. Er zerlegte die Zeichenkombination nach dem Gottesdeterminativ in DIŠ-ten $\hat{u}$, das alt mit AŠ gleichwertig ist (= gí ís $\left./ \mathrm{i}_{4}\right)$ und DAR. Die so resultierende Form des angeblichen DAR ist nun gegenüber der sonst weithin in zeitlich vergleichbaren Quellen bezeugten (MUŠEN-gunû: 7) ziemlich unglaubwürdig: Hinzu kommt, daß der erstere Beleg die Zeichen durch einen deutlichen Zwischenraum in $1+$ und (t) gliedert: ersteres ist ein BA ganz in der Art, wie es die EblaTexte schreiben; letzteres ist HुI $\times$ MAš, das aus Ebla mit dem Wert sur ${ }_{\mathrm{x}}$ bekannt ist ${ }^{9}$. Der somit wahrscheinlich zu lesende $\mathrm{GN}{ }^{\mathrm{d}} \mathrm{Ba}$ sur $-r a-a t$ ist allerdings $\mathrm{m}$. W. sonst nicht bezeugt.

5. „Dub-la“ (M. 2278 Z. 1: MAM 3, Nr. 12 [S. 319 f.]; BraunHolzinger S. 70 f.)

Das erste Zeichen ist nicht DUB, sondern deutlich GUL, wie es auch in Ebla üblich ist. Der somit zu lesende PN Gul-la kommt dort ebenfalls vor ${ }^{10}$.

6. IGI.ME (M. 2278 Z. 6, M. 2623 Z. 1'; MAM 3 Nr. 12 [S. 319 f.] bzw. Nr. 23 [S. 326]; Braun-Holzinger S. 70f.)

In beiden Fällen handelt es sich um die Wendung IGI.ME GN Ì.GUB, worin IGI.ME offenbar als Präposition fungiert. Das Logo-

S. z.B. ARET 3, S. 289.

${ }^{6}$ Ebd. sowie MEE 2, S. 347.

ARET 3, S. 274.

MEE 2, S. 337.

MEE 3, S. 198 Z. 66: HुI $\times$ MAš $=s u-u_{12}$-um („Sillabario di Ebla“).

10 MEE 2, S. 341; ARET 3, S. 274. 
gramm kommt in derselben Wendung auch aAK vor, und zwar mit syllabischer Verbalform: DÙL-su ša KÙ.AN IGI.ME ${ }^{\mathrm{d}} E n$-líl $i$-za-az "seine Statue aus . . .-Metall steht vor Enlil" ${ }^{\text {11 }}$. Lexikalisch ist IGI.ME in Ebla mit der Gleichung gi-ti-ma-a ${ }^{12}$ bezeugt, was sicherlich zur Wurzel $q-d-m$ gehört und wohl als /qidmay/ zu interpretieren ist: vgl. von derselben Wurzel akk. qudmu „Vorderseite“, qadmu „vorderer“. In präpositionaler Verwendung ist die Wurzel ug., aram., asa. und im Ge'ez üblich. Möglicherweise repräsentiert ME im Logogramm als phonetisches Komplement die monophthongisierte Form des in Ebla noch als Diphthong erhaltenen Suffixes /-ay/, also IGI-me.

7. „Mešgirru“ (M. 2341 Z. 1: MAM 3, Nr. 7 [S. 315]; BraunHolzinger, S. 70)

Dossin hatte als PN „Meš-gir ${ }_{10}$-ru“ mit vorangehender Präposition a-na gelesen. Da dann jedoch der Name des Stifters fehlt, mußte er als solchen denselben Namen voraussetzen: „Pour M. . . . (M.) a voué". Diese Struktur ist sehr unwahrscheinlich. BraunHolzinger zieht daher mit Recht $a-n a-z u$ dem PN. Ferner liest sie richtig URUDU anstelle von MES. NE.RU dürfte schließlich, wie Braun-Holzinger zu M. 2375 (s.u.) bemerkt, auch hier ein Syllabogramm darstellen, wie es sowohl in Ebla wie auch aAK bezeugt ist: rím/rim. Wählen wir nun noch für URUDU als syllabische Lesung $d a_{5}$, so ergibt sich der PN $A-n a-d a_{5}-$ rim, wozu $A-n a-d[a]^{?}-$ rí $^{\prime}$ aus Kišs $^{13}$ zu vergleichen wäre. In Ebla scheint der für URUDU übliche syllabische Wert allerdings $d a b_{6} \mathrm{zu} \operatorname{sein}^{14}$, woraus hier aber keine lexikalisch befriedigende Lesung resultieren würde.

8. „Tagge"-Inschrift (M. 2350: MAM 3, Nr. 10 [S. 317f.]; bei Braun-Holzinger, S. 70, nicht transkribiert)

$\mathrm{Ob}$ das in Z. 1 und Z. 7 stehende Gebilde, von Dossin als PN Tag - e $_{14}$ gelesen, ein Zeichen ( $\left.\mathrm{TAG}^{\prime}\right)$ oder eine Kombination von TAG mit einem zweiten Zeichen darstellt, ist noch unklar ${ }^{15}$ ebenso wie der Sinn der ganzen Inschrift, wenn man von NI-na BE-

11 AfO 20 (1963) 68, Rīmuš b 12 XXVII 12'-14'. Man würde statt $z a$ (Akk.) nominativisches $s$ u erwarten oder aber eine transitive Verbalform "stellte auf".

12 MEE 4, 0128 (S. 357). [Jetzt a. P. Steinkeller, OrAnt. 23 (1984) 33-7.]

${ }^{3}$ MAD 5, Nr. 45 II 8; von I. J. Gelb schon in MAD 3, S. 313, zitiert.

14 Vgl. Verf., ZA 72, 205.

$15 \mathrm{Vgl}$. zu dem Graphem und einer möglichen Entsprechung in Ebla D. O. Edzard, Le soi-disant "Tagge» et questions annexes, demnächst in MARI 4. 
li-sù „seinem Herrn“ in der II. Kolumne absieht. Hier sei lediglich darauf hingewisen, daß das reduplizierte Zeichen in Z. 5 nicht, wie von Dossin angenommen, HÚL (nach Dossins Umschrift HUUL) ist, sondern GIDIM oder das zur fraglichen Zeit davon nicht unterschiedene UDUG. Die "Totengeister" könnten somit das Stichwort für den Inhalt unserer Inschrift darstellen. Dazu würde eine Interpretation von me-da in der vorangehenden $\mathrm{Z}$. als Form von mîtum "tot" stimmen.

9. „Maššigirru“ (M. 2375 Z. 1; MAM 3, Nr. 5 S. 313; BraunHolzinger S. 70)

NE.RU ist, wie bereits Braun-Holzinger bemerkt, mim/rim. Das erste Zeichen sieht laut Kopie wie GÁ $\times$ AS̆ aus, weshalb Dossin „MAŠ(=MA[gemeint:MÀ]+A ̌s)-ši-gir $r_{10}-r u^{\prime \prime}$ liest. Sollte es sich um bloßes GÁ mit Kratzer handeln, so wäre $\mathrm{Ba}_{4}$-ši-rúm zu lesen. Aller-

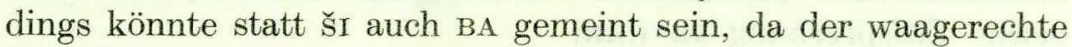
Keil deutlich im Inneren des Zeichens ansetzt.

10. Arši-aha (M. 2447 Z. 1: MAM 3, Nr. 13 [S. 320]; BraunHolzinger, S. 71).

Der fehlkopierte und nicht transkribierte PN in der ersten Zeile ist nach der Photographie MAM 3, S. 54, mit Sicherheit Ar-si-a-ha zu lesen; der Name ist auch in Ebla gut bezeugt ${ }^{16}$.

11. „Salim“ (M. $2306+2434+2390+2396$ Z. 1: MAM 3, Nr. 3 [S. 311]; Braun-Holzinger, S. 69)

Das als IGI $=1 \mathrm{im}$ interpretierte Zeichen ist der Form nach eher ein BA, wie der Vergleich mit IGI in den oben unter Nr. 6 angeführten Belegen zeigt. Der somit Sá-ba zu lesende PN ist auch in Ebla bezeugt ${ }^{17}$.

MEE 2, S. 337; ARET 3, S. 262. Auch aAK, s. MAD 3, S. 236.

17 MEE 2, S. 350; TM. 75. G. 1353 VIII 8 (OrAnt. 20 [1981] 40=StEb. 3 [1980] 13).

Es handelt sich jeweils um dieselbe Person.] 